\title{
Nonsemisimple Hopf algebras of dimension $8 p$ with the Chevalley property
}

\author{
Margaret Beattie, Gastón A. García, Siu-Hung Ng, and Jolie Roat \\ Abstract. Let $p$ be an odd prime and $\mathbb{k}$ an algebraically closed field of characteristic zero. We classify \\ nonsemisimple Hopf algebras over $\mathbb{k}$ of dimension $8 p$ with the Chevalley property, and give partial results \\ on nonsemisimple Hopf algebras of dimension 24.
}

\section{Introduction}

It was conjectured by Kaplansky $[\mathbf{K}]$ that there are only finitely many isomorphism classes of finitedimensional Hopf algebras over $\mathbb{k}$ for any given dimension. This conjecture was shown to be false by counterexamples of dimension $p^{4}$ with $p$ an odd prime [AS1, BDG, Ge2], but it is true for dimension 16 [GV]. However, the module categories over Hopf algebras of dimension 32 yield infinitely many inequivalent finite tensor categories $[\mathbf{E G}]$. In particular, there are infinitely isomorphism classes of Hopf algebras of dimension 32, see [B], [Gr3].

In the past two decades, Hopf algebras in a dimension which is factorized with few primes have been classified (cf. [BG2]). As a consequence, the number of isomorphsim classes of Hopf algebras of dimensions less than 32 but not equal to 24 is known to be finite. Semisimple Hopf algebras of dimension 24 with a $C^{*}$-structure were classified in $[\mathbf{I K}]$, but the general classification without $C^{*}$-structure remains open. In the nonsemisimple case, pointed Hopf algebras of dimension 24 are classified and there are finitely many of them up to isomorphism. However, not all the 24-dimensional Hopf algebras are pointed.

The family of Hopf algebras with the Chevalley property consists of Hopf algebras whose coradical forms a Hopf subalgebra. For example, pointed Hopf algebras have the Chevalley property. In fact, there exist $8 p$-dimensional Hopf algebras with the Chevalley property which are not pointed for each odd prime $p$, see Proposition 3.14. In this paper, in addition to some new general results, we will provide a complete classification of nonsemisimple Hopf algebras of dimension $8 p$ with $p$ an odd prime, which have the Chevalley property. Even though this result brings us one step closer to the complete classification of 24-dimensional nonsemisimple Hopf algebras, it remains unknown whether there exists any Hopf algebra $H$ of dimension 24 such that neither $H$ nor $H^{*}$ has the Chevalley property. Nevertheless, some new observations of 24dimensional Hopf algebras are presented in the last section of the paper which could be essential to the complete classification.

The paper is organized as follows. In Section 2 we introduce several definitions and conventions that will be needed along the paper. We also provide some descriptions on Hopf algebras with respect to their coalgebra structure and study pointed Hopf algebras over the group given by the semidirect product $\Gamma_{4 p}=C_{p} \rtimes_{\chi} C_{4}$. In Section 3 we collect some facts on nonsemisimple Hopf algebras of dimension $8 p$ and prove our main result in Theorem 3.16. The last section is devoted to the study of nonsemisimple Hopf algebras of dimension 24 .

2010 Mathematics Subject Classification. 16T05.

The second author was partially supported by ANPCyT-Foncyt, CONICET, and Secyt (UNLP). The third author was partially supported by the NSF grant DMS 1501179. 


\section{Preliminaries}

2.1. Definitions and Conventions. We work over an algebraically closed field $\mathbb{k}$ of characteristic zero. Let $H$ be a finite-dimensional Hopf algebra over $\mathbb{k}$ with antipode $\mathcal{S}$. We use Sweedler's notation $\Delta(h)=h_{1} \otimes h_{2}$ for the comultiplication in $H$, but dropping the summation symbol, see [S]. The readers are referred to [Mo] for details on the basic definitions of Hopf algebras.

The coradical $H_{0}$ of $H$ is the sum of all simple subcoalgebras of $H$. In particular, if $G(H)$ denotes the group of group-like elements of $H$, we have $\mathbb{k} G(H) \subseteq H_{0}$. We write $H_{0}=\bigoplus_{d \geq 1} H_{0, d}$, where $H_{0, d}$ denotes the direct sum of the simple subcoalgebras of $H$ of dimension $d^{2}$. By the Nichols-Zoeller Theorem, its dimension is divisible by the order of the group of group-like elements (see $[\mathbf{A N}]$ ).

We say that a Hopf algebra $H$ is pointed if $H_{0}=\mathbb{k} G(H)$, and it is called basic if all the simple $H$-modules are 1-dimensional. Since $H$ is finite-dimensional, $H$ is basic if and only if $H^{*}$ is pointed.

We denote by $\left\{H_{i}\right\}_{i \geq 0}$ the coradical filtration of $H$. If $H_{0}$ is a Hopf subalgebra of $H$, then

$$
\operatorname{gr} H=\bigoplus_{n \geq 0} \operatorname{gr} H(n)
$$

is the associated graded Hopf algebra, where gr $H(n)=H_{n} / H_{n-1}$ for $n \geq 0$ and $H_{-1}=0$. Let $\pi: \operatorname{gr} H \rightarrow H_{0}$ be the homogeneous projection. Then

$$
R=(\operatorname{gr} H)^{\operatorname{co} \pi}:=\{r \in \operatorname{gr} H \mid(\operatorname{id} \otimes \pi) \Delta(r)=r \otimes 1\}
$$

is called the diagram of $H$, which is a braided Hopf algebra in the category ${ }_{H_{0}}^{H_{0}} \mathcal{Y D}$ of left Yetter-Drinfeld modules over $H_{0}$, and it is a graded subalgebra of gr $H$. The linear subspace $R(1)$ of homogeneous elements of $R$ of degree one, with the braiding from ${ }_{H_{0}}^{H_{0}} \mathcal{Y D}$, is called the infinitesimal braiding of $H$ and coincides with the subspace of primitive elements

$$
\mathcal{P}=\left\{r \in R \mid \Delta_{R}(r)=r \otimes 1+1 \otimes r\right\},
$$

where $\Delta_{R}$ denotes the comultiplication of $R$. It turns out that the Hopf algebra gr $H$ is the Radford biproduct (or Magid bosonization) gr $H \simeq R \# H_{0}$ (cf. [Ra, Ma]). The subalgebra of $R$ generated by $R(1)=V$ is isomorphic to the Nichols algebra $\mathfrak{B}(V)$. If $H_{0}=\mathbb{k} G$ is a group algebra, we denote the category of YetterDrinfeld modules simply by ${ }_{G}^{G} \mathcal{Y D}$. Specifically, a left Yetter-Drinfeld module over $\mathbb{k} G$ is a finite-dimensional $G$-graded $\mathbb{k}$-linear space $M=\oplus_{h \in G} M_{h}$ with a left $\mathbb{k} G$-module $(M, \cdot)$ structure such that

$$
\delta(g \cdot m)=g h g^{-1} \otimes g \cdot m, \quad \forall m \in M_{h}, g, h \in G .
$$

Here $\delta$ is left $\mathbb{k} G$-comodule structure on $M$ given by the $G$-grading, namely with $\delta(m)=h \otimes m$ for $m \in M_{h}$.

Yetter-Drinfeld modules over $G$ are completely reducible and the irreducible ones are parametrized by pairs $(\mathcal{O}, \rho)$, where $\mathcal{O}$ is a conjugacy class of $G$ and $(\rho, V)$ is an irreducible representation of the centralizer $C_{G}(\sigma)$ of a fixed element $\sigma \in \mathcal{O}$ : Then the irreducible Yetter-Drinfeld module $M(\mathcal{O}, \rho)$ is the induced $\mathbb{k} G$ module $\mathbb{k} G \otimes_{\mathbb{k} C_{G}(\sigma)} V$. Let $1=g_{1}, \ldots, g_{n}$ be a complete set of left coset representatives of $C_{G}(\sigma)$ in $G$. Then $\sigma_{i}=g_{i} \sigma g_{i}^{-1}, i=1, \ldots, n$, are all the elements of $\mathcal{O}$ and $M(\mathcal{O}, \rho)=\bigoplus_{i \leq 1 \leq n} g_{i} \otimes V$. The $\mathbb{k} G$-comodule structure on $M(\mathcal{O}, \rho)$ is given by $\delta\left(g_{i} v\right)=\sigma_{i}$ for all $i=1, \ldots, n$, where $g_{i} v$ denotes the element $g_{i} \otimes v \in g_{i} \otimes V$. In particular, $\operatorname{dim} M(\mathcal{O}, \rho)=|\mathcal{O}| \operatorname{dim} V$. We denote the corresponding Nichols algebra by $\mathfrak{B}(\mathcal{O}, \rho)$. For the definition of Nichols algebras and basic facts, we refer the reader to $[\mathbf{A}]$.

Throughout this paper, the cyclic group of order $\ell$ is denoted by $C_{\ell}$. For $n \in \mathbb{N}, n \geq 3$, the dihedral group of order $2 n$ is denoted by $\mathbb{D}_{n}$; we will use the presentation

$$
\left.\mathbb{D}_{n}=\langle a, y| a^{2}=y^{n}=1, \text { aya }=y^{-1}\right\rangle .
$$

The dicyclic group of order $4 n$, denoted by $\mathrm{Dic}_{n}$, has the presentation

$$
\operatorname{Dic}_{n}=\left\langle x, y \mid x^{4}=y^{n}=1, x y x^{-1}=y^{-1}\right\rangle .
$$

Let $N$ be a positive integer and let $q \in \mathbb{k}$ be a primitive $N$-th root of unity. The Taft algebra $T_{q}$ is the $\mathbb{k}$-algebra generated by the elements $g$ and $x$ satisfying the relations $x^{N}=0=1-g^{N}, g x=q x g$. It is a Hopf algebra with its coalgebra structure determined by $g$ being group-like and $x$ a $(1, g)$-primitive, i.e., $\Delta(g)=g \otimes g$ and $\Delta(x)=x \otimes 1+g \otimes x$. It is self-dual and pointed of dimension $N^{2}$. If $N=2$ so that $q=-1$, then $T_{-1}$ is called the Sweedler algebra and will be denoted by $H_{4}$ throughout this article. 
Recall that a tensor category $\mathcal{C}$ over $\mathbb{k}$ has the Chevalley property if the tensor product of any two simple objects is semisimple. We shall say that a Hopf algebra $H$ has the Chevalley property if the category Comod $(H)$ of $H$-comodules does. In particular, $H$ has the Chevalley property if and only if the coradical is a Hopf subalgebra.

REMARK 2.1. In some literature (for example $[\mathbf{N N}]$ ), $H$ is said to have the Chevalley property if $H$-mod does. In this case, $H$ has the Chevalley property if the Jacobson radical of $H$ is a Hopf ideal.

2.2. On simple coalgebras, group-likes and skew-primitives. In this subsection we collect some structural results that will be needed later. by

Recall that $H$ is a free left and a free right $H^{*}$-module of rank 1 via the $H^{*}$-actions $\rightarrow$ and $<$ defined

$$
f \rightarrow h=f\left(h_{2}\right) h_{1} \quad \text { and } \quad h<f=f\left(h_{1}\right) h_{2}, \quad \text { for all } f \in H^{*} \text { and } h \in H .
$$

Thus, a finite-dimensional $\mathbb{k}$-linear space $V$ is a right (resp. left) $H$-comodule if and only if $V$ is a left (resp. right) $H^{*}$-module. If $\sigma$ is an algebra automorphism of $H^{*}$, then $V$ can be turned into another left $H^{*}$-module ${ }_{\sigma} V$ with the $H^{*}$-action twisted by $\sigma$, namely

$$
f \cdot v:=\sigma(f) v, \quad \text { for } f \in H^{*} \text { and } v \in V .
$$

Lemma 2.2. Let $C$ be a 4-dimensional simple coalgebra over $\mathbb{k}$ and $T: C \rightarrow C$ a coalgebra automorphism with finite order. If $\operatorname{Tr}(T)=0$, then $\operatorname{ord}(T)=2$.

Proof. Let $n=\operatorname{ord}(T)$. By [S997, Theorem $1.4(\mathrm{~b})]$, there is a primitive $n$-th root $\omega \in \mathbb{k}$ and a basis $\left\{e_{i j}\right\}_{i, j=1,2}$ of $C$ such that $T\left(e_{i j}\right)=\omega^{i-j} e_{i j}$. Thus, $\operatorname{Tr}(T)=(1+\omega)\left(1+\omega^{-1}\right)$ which is zero, if and only if, $\omega=-1$. Therefore, $n=2$.

Lemma 2.3. Let $H$ be a finite-dimensional Hopf algebra that admits a decomposition of coalgebras $H=C \oplus C^{\prime}$ with $C$ simple. Then $C$ is invariant under $\mathcal{S}^{-2} \circ L(a)$, where $a$ is the distinguished group-like element of $H$ and $L(a)$ denotes the left multiplication operator on $H$ by $a$. Moreover, $\operatorname{Tr}_{C}\left(\mathcal{S}^{-2} \circ L(a)\right)=0$ provided $a$ is nontrivial. If, in addition, $\operatorname{dim} C=4$, then $\left.\mathcal{S}^{4}\right|_{C}=\left.L\left(a^{2}\right)\right|_{C}$.

Proof. Let $I$ be a simple right coideal of $H$ such that $\Delta(I) \subset I \otimes C$. Then $C \cong \operatorname{dim}(I) I$ as right $H$ comodules and hence left $H^{*}$-modules. In particular, $I$ is a simple projective $H^{*}$-module. By $[\mathrm{Ng}$, Lemma 1.1], we have that $I \cong \mathbb{k}_{\widehat{a^{-1}}} \otimes I^{\vee \vee}$, where $I^{\vee}$ denotes the left dual of $I$ in the category of finite-dimensional $H^{*}$-modules, and $\hat{x} \in H^{* *}$ denotes the image of $x \in H$ under the natural isomorphism of $H \simeq H^{* *}$. In particular, $I \cong{ }_{\sigma} I$ where $\sigma=\left(\mathcal{S}^{*}\right)^{2} \circ R\left(\widehat{a^{-1}}\right)$ (cf. [Ng, Lemma 1.2]). Therefore, the annihilator ideal $\operatorname{ann}_{H^{*}} I$ is invariant under the automorphism $\sigma$. Note that $\sigma=\tau^{*}$ where $\tau=\mathcal{S}^{2} \circ L\left(a^{-1}\right)$ is a coalgebra automorphism. Since $\operatorname{ann}_{H^{*}} I=C^{\perp}$, we find $\sigma\left(C^{\perp}\right)=C^{\perp}$ and hence $C$ is invariant under $\tau$.

Since $I \cong{ }_{\sigma} I$ as $H^{*}$-modules, $C \cong{ }_{\sigma} C$ as $H^{*}$-modules. To complete the proof, by [ $\mathrm{Ng}$, Lemma 1.2], it suffices to show that $\tau^{-1}: C \rightarrow{ }_{\sigma} C$ is a $H^{*}$-module homomorphism. For $f \in H^{*}$ and $c \in C$,

$$
\sigma(f) \rightarrow \tau^{-1} c=\sigma(f)\left(\tau^{-1} c_{2}\right) \tau^{-1} c_{1}=f\left(\tau\left(\tau^{-1} c_{2}\right)\right) \tau^{-1} c_{1}=f\left(c_{2}\right) \tau^{-1} c_{1}=\tau^{-1}(f \rightarrow c) .
$$

Therefore, $\tau^{-1} \in \operatorname{Hom}_{H^{*}}\left(C,{ }_{\sigma} C\right)$. The last assertion follows then by Lemma 2.2.

Lemma 2.4. Let $H$ be a finite-dimensional Hopf algebra and $A$ a Hopf subalgebra. Suppose $H$ admits a subcoalgebra decomposition $H=A \oplus C$ for some subcoalgebra $C$ of $H$. Then $H$ and $A$ have a common distinguished group-like element.

Proof. Let $\lambda$ be a nonzero right integral of $H^{*}$. Then, for all $h \in H, h<\lambda=\lambda(h) 1$ and $\lambda \rightarrow h=\lambda(h) a$ where $a$ is the distinguished group-like element of $H$. Note that for $c \in C$, we have $\lambda(c)=0$. Otherwise, 
$\lambda(c) 1=\lambda\left(c_{1}\right) c_{2} \in C$ and hence $1 \in C$, which contradicts that $C \cap A=0$. Therefore, $\left.\lambda\right|_{A} \neq 0$ and it is a right integral of $A$. Let $h \in A$ such that $\lambda(h) \neq 0$. Then

$$
a=\lambda(h)^{-1} \lambda\left(h_{2}\right) h_{1} \in A .
$$

Thus, $a$ is the distinguished group-like element of $A$.

The following proposition is a refined version of [BG, Proposition 2.17] and [HN, Proposition 1.3].

Proposition 2.5. Let $\pi: H \rightarrow A$ be a Hopf algebra epimorphism and assume $\operatorname{dim} H=2 \operatorname{dim} A$. Then $H^{c o \pi}=\mathbb{k}\{1, x\}$ with $x$ a $(1, g)$-primitive element, $g \in G(H)$ and $2 \mid$ ord $g$. Moreover, if $x$ is a trivial skewprimitive, i.e., a nonzero scalar multiple of $1-g$, then ord $g=2$ and $H$ fits into an exact sequence of Hopf algebras $\mathbb{k} C_{2} \hookrightarrow H \rightarrow A$. If $x$ is nontrivial, then $x^{2}=0$ and $H$ contains a Hopf subalgebra of dimension $2 \operatorname{ord}(g)$; in particular, $4 \mid \operatorname{dim} H$.

2.3. On pointed Hopf algebras over $\Gamma_{4 p}$. Our main results will involve Hopf algebras whose coradical is a semisimple Hopf algebra of dimension $4 p$ for $p$ an odd prime. To this end, we need some results on the simple representations of the group $\Gamma_{4 p}$. Recall that for $p$ a prime congruent to $1 \bmod 4, \Gamma_{4 p}$ is defined to be the group given by the semidirect product $\Gamma_{4 p}=C_{p} \rtimes_{\chi} C_{4}$ where $C_{p}=\langle y\rangle, C_{4}=\langle x\rangle$ are the cyclic groups of order $p$ and 4 , respectively, and $\chi(x)(y)=y^{\ell}$, where $\ell=\frac{p-1}{2}$. Moreover, $\Gamma_{4 p}$ has the presentation

$$
\Gamma_{4 p}=\left\langle x, y: x^{4}=1=y^{p}, x y=y^{\ell} x\right\rangle,
$$

and it has $4+\frac{p-1}{4}$ conjugacy classes and hence $\Gamma_{4 p}$ has $4+\frac{p-1}{4}$ simple representations. Among them, 4 are one-dimensional, say $\alpha_{0}, \ldots, \alpha_{3}$, which are given the quotient $\Gamma_{4 p} / C_{p}$. The remaining $\frac{p-1}{4}$ simple representations are 4-dimensional, say $\left(\beta_{1}, V_{1}\right), \ldots,\left(\beta_{\frac{p-1}{4}}, V_{\frac{p-1}{4}}\right)$.

Denote by $\omega$ a primitive 4 th root of unity and by $q$ a primitive $p$ th root of unity.

\begin{tabular}{|l|l|l|l|}
\hline Conjugacy class $\mathcal{O}$ & Centralizer & Irrepn. $\rho$ & $\operatorname{dim} M(\mathcal{O}, \rho)$ \\
\hline$e$ & $\Gamma_{4 p}$ & $\alpha_{j}, 0 \leq j \leq 3$ & 1 \\
\cline { 3 - 4 } & & $\beta_{j}, 1 \leq j \leq \frac{p-1}{4}$ & 4 \\
\hline $\begin{array}{l}\mathcal{O}_{x^{m}}=\left\{x^{m}, y x^{m}, \ldots, y^{p-1} x^{m}\right\}, \\
\left|\mathcal{O}_{x^{m}}\right|=p, 0<m<4\end{array}$ & $\langle x\rangle \simeq C_{4}$ & $\begin{array}{l}\chi_{j}, \chi_{j}(x)=\omega^{j} \\
0 \leq j \leq 3\end{array}$ & $p \geq 5$ \\
\hline $\mathcal{O}_{y^{k}}=\left\{y^{k}, y^{k \ell}, y^{k \ell^{2}}, y^{k \ell^{3}}\right\}$, & $\langle y\rangle \simeq C_{p}$ & $\psi_{s}, \psi_{s}(y)=q^{s}$ & 4 \\
$\left|\mathcal{O}_{y^{k}}\right|=4,1<k \leq \frac{p-1}{4}$ & & $0 \leq s<p$ & \\
\hline
\end{tabular}

TABLE $1 . \Gamma_{4 p}$, with $p \equiv 1 \bmod 4$.

Lemma 2.6. Assume either $\mathcal{O}$ is trivial or $\mathcal{O}=\mathcal{O}_{y^{k}}$ for $1<k \leq \frac{p-1}{4}$. Then $\operatorname{dim} \mathfrak{B}(\mathcal{O}, \rho)$ is infinite for $\rho$ any simple representation of $\Gamma_{4 p}$ or simple representation of $C_{p}$, respectively.

Proof. Assume first that $\mathcal{O}=\{e\}$ and let $(\rho, V)$ be a simple representation of $\Gamma_{4 p}$. Then $M(\mathcal{O}, \rho)=V$ is the $\mathbb{k} G$-module $V$ with the trivial $G$-grading. This implies that $c(v \otimes w)=w \otimes v$ for all $v, w \in V$; in particular, $c(v \otimes v)=v \otimes v$. Consequently, $\operatorname{dim} \mathfrak{B}(\mathcal{O}, \rho)$ is infinite, since it contains the polynomial algebra in $v$.

Assume now that $\mathcal{O}=\mathcal{O}_{y^{k}}=\left\{y^{k}, y^{k \ell}, y^{k \ell^{2}}, y^{k \ell^{3}}\right\}=\left\{y^{k}, x y^{k} x^{-1}, x^{2} y^{k} x^{-2}, x^{3} y^{k} x^{-3}\right\}$ for some $k \neq 0$. Since $C_{\Gamma_{4 p}}\left(y^{k}\right)=\langle y\rangle \simeq C_{p}$, all simple representations are one-dimensional, say $\psi_{0}, \ldots, \psi_{p-1}$ with $\psi_{s}(y)=q^{s}$. Then $M\left(\mathcal{O}_{y^{k}}, \psi_{s}\right)=\bigoplus_{j=0}^{3} x^{j} \otimes \mathbb{k} 1$. If we denote $w_{j}=x^{j} \otimes 1$, we have that

$$
x \cdot w_{j}=w_{j+1}, \quad y \cdot w_{j}=q^{s \ell^{4-j}} w_{j}, \quad \text { and } \quad \delta\left(w_{j}\right)=y^{k \ell^{j}} \otimes w_{j} \quad \text { for all } j \in \mathbb{Z}_{4} .
$$

In particular, the formula for the braiding equals

$$
c\left(w_{r} \otimes w_{t}\right)=y^{k \ell^{r}} \cdot w_{t} \otimes w_{r}=q^{k s \ell^{r+4-t}} w_{t} \otimes w_{r} \quad \text { for all } 0 \leq r, t<4 ;
$$


that is, $M\left(\mathcal{O}_{y^{k}}, \psi_{s}\right)$ is a braided vector space of diagonal type. If $s=0$, then $c\left(w_{r} \otimes w_{t}\right)=w_{t} \otimes w_{r}$, which implies that $\operatorname{dim} \mathfrak{B}\left(\mathcal{O}_{y^{k}}, \psi_{0}\right)=\infty$. Assume $s \neq 0$ and denote $\xi=q^{k s}$. Then by [H, Theorem 17], we have that $\operatorname{dim} \mathfrak{B}\left(\mathcal{O}_{y^{k}}, \psi_{s}\right)=\infty$ for all $0<s<p$.

REMARK 2.7. For $0<m<4$, consider the conjugacy class $\mathcal{O}=\mathcal{O}_{x^{m}}=\left\{x^{m}, y x^{m} y^{-1}, \ldots, y^{p-1} x^{m} y^{1-p}\right\}$, and let $\chi_{k}$ be the one-dimensional representation of $\Gamma_{4 p}$ given by $\chi_{k}(x)=\omega^{k}$ for $0<k<4$. Then, $M\left(\mathcal{O}_{x^{m}}, \chi_{k}\right)=\bigoplus_{j=0}^{p-1} y^{j} \otimes \mathbb{k} 1$; in particular, $\operatorname{dim} M\left(\mathcal{O}_{x^{m}}, \chi_{k}\right)=p$. If we write $v_{j}=y^{j} \otimes 1$ for $0 \leq j<p$, we have that

$$
x \cdot v_{j}=\omega^{k} v_{j \ell+1}, \quad y \cdot v_{j}=v_{j+1}, \quad \text { and } \quad \delta\left(v_{j}\right)=y^{j\left(1-\ell^{m}\right)} x^{m} \otimes v_{j} \quad \text { for all } j \in \mathbb{Z}_{p} .
$$

In particular, the braiding equals

$$
c\left(v_{r} \otimes v_{t}\right)=y^{r\left(1-\ell^{m}\right)} x^{m} \cdot v_{t} \otimes v_{r}=\omega^{m k} v_{t \ell^{m}+r\left(1-\ell^{m}\right)} \otimes v_{r} \quad \text { for all } 0 \leq r, t<p .
$$

Consequently, if $m k \equiv 0 \bmod 4$, we have that $c\left(v_{0} \otimes v_{0}\right)=x^{m} \cdot v_{0} \otimes v_{0}=v_{0} \otimes v_{0}$, which implies that $\operatorname{dim} \mathfrak{B}\left(\mathcal{O}_{x^{m}}, \chi_{k}\right)=\infty$. If $m k \not \equiv 0 \bmod 4$, pointed Hopf algebras with group of points $\Gamma_{4 p}$ do occur, see [Gr4], [AG].

\section{Nonsemisimple Hopf algebras $H$ of dimension $8 p$}

Throughout this section, we assume that $H$ is a nonsemisimple Hopf algebra of dimension $8 p$, with $p$ an odd prime unless specified otherwise. For each $\beta \in G\left(H^{*}\right)$, the 1-dimensional $H$-module associated with $\beta$ is denoted by $\mathbb{k}_{\beta}$. In particular, $\mathbb{k}_{\varepsilon}$ is the trivial 1-dimensional $H$-module. We denote the Jacobson radical of $H$ by $J(H)$, and the projective cover of an $H$-module $V$ by $P(V)$.

3.1. Nonsemisimple Hopf algebras $H$ of dimension $8 p$ with $|G(H)|=2 p$. In this subsection we improve upon the results of [BG2] for $H$ a nonsemisimple nonpointed Hopf algebra of dimension $8 p$ and group likes of order $2 p, p$ an odd prime. We begin by recalling the well-known structure of the pointed Hopf algebras of dimension $4 p$.

REMARK 3.1. For $p$ an odd prime, nonsemisimple pointed Hopf algebras of dimension $4 p$ were described in [AN, A.1] and for $p=2$ the pointed Hopf algebras of dimension 8 are well-known and were described for example in [Ş97]. In the first case, all such Hopf algebras $H$ have $G(H) \simeq C_{2 p}$. The pointed Hopf algebras of dimension 8 can have group-likes of order 2 or 4 and, if order 4 , can have group of group-likes isomorphic to the Klein 4 -group or to the cyclic group of order 4 . In either case ( $p$ odd or $p=2$ ), there are 4 nonisomorphic Hopf algebras with group-likes of order $2 p$ as listed below. Here $g, h$ are group-like elements, $x$ is skew-primitive and $\lambda$ is a primitive $2 p$ th root of unity.

$$
\begin{aligned}
\mathcal{A}(-1,0) & :=\mathbb{k}\left\langle g, x \mid g^{2 p}-1=x^{2}=g x+x g=0\right\rangle, \quad \Delta(x)=x \otimes 1+g \otimes x . \\
\mathcal{A}(-1,0)^{*} & :=\mathbb{k}\left\langle g, x \mid g^{2 p}-1=x^{2}=g x-\lambda x g=0\right\rangle, \quad \Delta(x)=x \otimes 1+g^{p} \otimes x . \\
\mathcal{A}(-1,1) & :=\mathbb{k}\left\langle g, x \mid g^{2 p}-1=x^{2}-g^{2}+1=g x+x g=0\right\rangle, \quad \Delta(x)=x \otimes 1+g \otimes x . \\
H_{4} \otimes \mathbb{k} C_{p} & :=\mathbb{k}\left\langle g, h, x \mid h^{2}-1=g^{p}-1=x^{2}=g h-h g=h x+x h=g x-x g=0\right\rangle, \quad \Delta(x)=x \otimes 1+h \otimes x .
\end{aligned}
$$

In the case $p=2$, we write $\mathcal{A}_{8}(-1,1)$ to emphasize it is 8 -dimensional. Note that $\mathcal{A}_{8}(-1,1)=A_{4}^{\prime \prime}$ in [S97] notation.

The dual of $\mathcal{A}(-1,1)$ is nonpointed and as a coalgebra is isomorphic to $H_{4} \oplus \mathcal{M}^{*}(2, \mathbb{k})^{p-1}$. On the other hand, the Hopf algebras $\mathcal{A}(-1,0)$ and $\mathcal{A}(-1,1)$ do not have Hopf subalgebras isomorphic to $H_{4}$ but $\mathcal{A}(-1,0)^{*}$ and $H_{4} \otimes \mathbb{k} C_{p}$ do. In all four cases, $\mathcal{S}^{4}=$ id. The distinguished group-like elements of $\mathcal{A}(-1,0)$, $\mathcal{A}(-1,0)^{*}$ and $\mathcal{A}(-1,1)$ are respectively $g, g$ and $g^{p}$ in the above convention.

The following is known from [BG2]. 
Proposition 3.2. [BG2, Propositions 4.5, 4.6, 4.7] Let $H$ be a nonsemisimple nonpointed Hopf algebra of dimension $8 p$ and group-likes of order $2 p, p$ an odd prime. Then:

(a) $H$ contains a pointed Hopf subalgebra $A$ of dimension $4 p$. As a coalgebra $H \simeq A \oplus \mathcal{M}^{*}(2, \mathbb{k})^{p}$ and $H_{0} \simeq \mathbb{k} C_{2 p} \oplus \mathcal{M}^{*}(2, \mathbb{k})^{p}$ and so has dimension $6 p$.

(b) $H^{*}$ does not have a Hopf subalgebra isomorphic to $H_{4}$.

(c) $\left|G\left(H^{*}\right)\right|$ is 2 or 4 .

(d) Suppose either

(i) $A^{*}$ is nonpointed (so that $A$ is isomorphic to $\mathcal{A}(-1,1)$ ), or

(ii) $A^{*}$ is pointed and $H^{*}$ has a nontrivial skew-primitive, then $H^{*}$ has a Hopf subalgebra isomorphic to $\mathcal{A}_{8}(-1,1)$.

Now we strengthen the results above.

Lemma 3.3. Let $H$ be an $8 p$-dimensional nonsemisimple Hopf algebra with $|G(H)|=2 p$ such that $H$ and $H^{*}$ are nonpointed. Then $H$ contains a pointed normal Hopf subalgebra $A$, isomorphic to either $\mathcal{A}(-1,1)$ or $\mathcal{A}(-1,0)$, and fits into the exact sequence $1 \rightarrow A \rightarrow H \rightarrow \mathbb{k} C_{2} \rightarrow 1$, which has the dual exact sequence $1 \rightarrow \mathbb{k} C_{2} \rightarrow H^{*} \stackrel{\pi}{\rightarrow} A^{*} \rightarrow 1$. Furthermore, $G\left(H^{*}\right) \simeq C_{4}$ and $H^{*}$ has a Hopf subalgebra $Y$ isomorphic to $\mathcal{A}_{8}(-1,1)$ with image in $A^{*}$ isomorphic to $H_{4}$.

Proof. Let $A$ be the Hopf subalgebra of $H$ given by Proposition $3.2(a)$, and write $\pi: H^{*} \rightarrow A^{*}$ for the induced surjection. By Proposition 2.5, $R=H^{* \operatorname{co} \pi}$ is either a group algebra of dimension 2 or it is generated by a nontrivial $(1, g)$-primitive $y$ with $y^{2}=0$ and $2 \mid \operatorname{ord}(g)$. Assume the latter case. As $R$ is left-normal, it follows that $h_{1} y \mathcal{S}\left(h_{2}\right) \in \mathbb{k} y$ for all $h \in H^{*}$. Thus, $h y=h_{1} y \mathcal{S}\left(h_{2}\right) h_{3}=c y k$ with $c \in \mathbb{k}$ and $k \in H^{+}$, which implies that $H^{*} y \subset y H^{*}$. Therefore, $y H^{*}=R^{+} H^{*}$ is a Hopf ideal of $H^{*}$ and $H^{*} / R^{+} H^{*} \cong A^{*}$. In particular, $\operatorname{dim} R^{+} H^{*}=4 p$. As $y^{2}=0$, it follows that $R^{+} H^{*} \subset J\left(H^{*}\right)$. But since $\operatorname{dim} H_{0}=6 p$, we have that $\operatorname{dim} J\left(H^{*}\right)=2 p$, a contradiction. Therefore, $R$ is a 2-dimensional normal Hopf subalgebra of $H^{*}$, and hence $A$ is a normal Hopf subalgebra of $H$.

Let $e_{0}, \ldots, e_{2 p-1}$ be the primitive idempotents of $K=\mathbb{k} G(H)$. Since $H$ is a free $K$-module, $\operatorname{dim} H e_{i}=4$. Therefore, $\operatorname{dim} P \leq 4$ for all principal projective $H$-modules $P$. Suppose $H^{*}$ has only trivial skew-primitive elements. Then $P(\mathbb{k})$ must have a simple constituent $V$ with $\operatorname{dim} V>1$. This implies $\operatorname{dim} P\left(V^{*}\right)>4$, giving a contradiction and so showing that $H^{*}$ has a nontrivial skew-primitive. Thus by Proposition $3.2(d), H^{*}$ contains a Hopf subalgebra $Y$ isomorphic to $\mathcal{A}_{8}(-1,1)$. By the structure of $\mathcal{A}_{8}(-1,1)$ and by Proposition $3.2(c), G\left(H^{*}\right) \simeq \mathbb{k} C_{4}$.

Write $1 \rightarrow \mathbb{k}\langle\beta\rangle \rightarrow H^{*} \rightarrow A^{*} \rightarrow 1$ with $\beta$ a group-like element of order 2. Suppose that $Y$ is generated by the $(1, \alpha)$-primitive $y$, where $\alpha$ has order 4 . Then $\beta=\alpha^{2}$ and $\pi(\beta)=1$. Thus the image of $G\left(H^{*}\right)$ under $\pi$ is $\mathbb{k} C_{2} \simeq \mathbb{k}\langle\pi(\alpha)\rangle$. Since $y \notin H^{* c o \pi}$, then $\pi(y) \neq 0$ so that $\pi(y)$ is a nontrivial $(1, \pi(\alpha))$-primitive. and $\pi\left(A_{8}(-1,1)\right)$ is isomorphic to $H_{4}$. Then $A^{*}$ must contain a Hopf subalgebra isomorphic to $H_{4}$, so that by Remark 3.1, $A$ is isomorphic to either $\mathcal{A}(-1,1)$ or $\mathcal{A}(-1,0)$.

We end this subsection with the following theorems.

Theorem 3.4. Let $H$ be an $8 p$-dimensional nonsemisimple Hopf algebra with $|G(H)|=2 p$ such that both $H$ and $H^{*}$ are nonpointed. Then $\operatorname{ord}\left(\mathcal{S}^{2}\right)=2 p$.

Proof. By Lemma 3.3 and Remark 3.1, $H$ contains a pointed $4 p$-dimensional Hopf subalgebra $A$ generated by a group-like element $g$ of order $2 p$ and a $(1, g)$-primitive element $x$, and $H=A \oplus H_{0,2}$ as coalgebras. Note that, $\left.\mathcal{S}^{4}\right|_{A}=\operatorname{id}_{A}$ and by Lemma 2.4, $g$ is the distinguished group-like element of $H$. Let $D$ be a simple subcoalgebra of dimension 4. By Lemmas 2.2 and 2.3, there exists a matrix basis $\left\{e_{11}, e_{22}, e_{21}, e_{12}\right\}$ of $D$ 
such that $\mathcal{S}^{2}\left(e_{i j}\right)=(-1)^{i-j} g e_{i j}$. Since $G(H)$ acts on the 4-dimensional simple subcoalgebras of $H, g^{p} D=D$ and so $\mathcal{S}^{2 p}(D)=D$. If $g D=D$, then $D$ is a left $(\mathbb{k}\langle g\rangle, H)$-Hopf module and hence $D$ must be free over $\mathbb{k}\langle g\rangle$. This is not possible since $\operatorname{dim} D=4$. Therefore, $g D \neq D$. In particular, $\mathcal{S}^{2}(D) \cap D=0$ and $p\left|\operatorname{ord}\left(\mathcal{S}^{2}\right)\right| 4 p$.

Suppose $\left.\mathcal{S}^{2 p}\right|_{D}=\mathrm{id}$. Then the subalgebra $K$ of $H$ generated by $\mathbb{k}\langle g\rangle \oplus D$ is a sub-bialgebra (and hence a Hopf subalgebra) of dimension $>4 p$. Therefore, $K=H$ and hence $\mathcal{S}^{2 p}=$ id. This implies $\left.\mathcal{S}^{2 p}\right|_{A}=$ id. However, since $A$ is a pointed nonsemisimple Hopf algebra of dimension $4 p, \operatorname{ord}\left(\left.\mathcal{S}^{2}\right|_{A}\right)=2$, a contradiction. Therefore, $\operatorname{ord}\left(\left.\mathcal{S}^{2}\right|_{H_{0,2}}\right)=2 p=\operatorname{ord}\left(\mathcal{S}^{2}\right)$.

Theorem 3.5. Let $H$ be a Hopf algebra of dimension $2^{n} p$ over $\mathbb{k}$, where $n \geq 0$ and $p$ is an odd prime. If $H$ has a semisimple Hopf subalgebra of dimension $2^{n}$, then $H$ is semisimple.

Proof. Suppose $H$ has a semisimple Hopf subalgebra $K$ of dimension $2^{n}$, and let $\mathcal{S}$ be the antipode of $H$. We consider three cases to show that $H$ must be semisimple.

First, suppose that $H$ contains a group-like element $a$ of order $p$. Define $\bar{K}$ to be the $\mathbb{k}$-subalgebra of $H$ generated by $C=K \oplus \mathbb{k}\langle a\rangle$. Then, $\bar{K}$ is a Hopf subalgebra and $\operatorname{dim} \bar{K}>2^{n}$, and so $\bar{K}=H$. Further, since $C$ is the direct sum of two semisimple Hopf algebras as a vector space, $\left.\mathcal{S}^{2}\right|_{C}=\mathrm{id}_{C}$ and so $S^{2}=\mathrm{id}_{H}$. Hence $H$ must be semisimple.

Next suppose that $H^{*}$ contains a group-like element $\alpha$ of order $p$. Let $\pi: H^{*} \rightarrow K^{*}$ be the Hopf algebra surjection dual to the inclusion of $K$ in $H$. Since $\operatorname{ord}(\pi(\alpha)) \mid \operatorname{dim} K, \pi(\alpha)=1$ and so $\alpha \in\left(H^{*}\right)^{\operatorname{co} \pi}$. Thus $\mathbb{k}\langle\alpha\rangle \subset\left(H^{*}\right)^{\operatorname{co} \pi}$. However, since $\operatorname{dim}\left(H^{*}\right)^{\operatorname{co} \pi}=p$, we actually have $\mathbb{k}\langle\alpha\rangle=\left(H^{*}\right)^{\operatorname{co} \pi}$ and thus the exact sequence

$$
1 \rightarrow \mathbb{k} C_{p} \rightarrow H^{*} \rightarrow K^{*} \rightarrow 1
$$

of Hopf algebras. Since $\mathbb{k} C_{p}$ and $K^{*}$ are semisimple, so is $H^{*}$ and thus so is $H$.

Finally suppose that neither $H$ nor $H^{*}$ has any group-like element of order $p$. By Radford's formula, we have $\left(\mathcal{S}^{4}\right)^{2 n}=\operatorname{id}_{H}$. In particular, $\operatorname{ord}\left(\mathcal{S}^{2}\right)$ is a 2-power. Let $e$ be the normalized integral of $K$. Since $H$ is a free right $K$-module, $\operatorname{dim} H e=p$.

Suppose $H$ is not semisimple. It follows from [RS, Lemma 3] that $\operatorname{Tr}\left(\mathcal{S}^{2} \circ R(e)\right)=0$ and hence $\operatorname{Tr}\left(\left.\mathcal{S}^{2}\right|_{H e}\right)=0$. However, we know $\operatorname{dim} H e=p$ and the order of $\mathcal{S}^{2}$ is a 2-power, so by [HN, Lemma 1.8], $\operatorname{Tr}\left(\left.\mathcal{S}^{2}\right|_{H e}\right) \neq 0$, a contradiction! Therefore, $H$ is semisimple.

3.2. Nonsemisimple Hopf algebras of dimension $8 p$ with the Chevalley Property. Throughout this subsection, we assume that $H$ is a Hopf algebra of dimension $8 p, p$ an odd prime. If $H$ has the Chevalley property, by the Nichols-Zoeller Theorem, the dimension $n$ of $H_{0}$ must be a divisor of $8 p$, i.e., $n \in\{2,4,8, p, 2 p, 4 p, 8 p\}$. If $H$ is not pointed, since the only semisimple Hopf algebras of dimension $2,4, p$ are group algebras, it follows that $n \in\{8,2 p, 4 p\}$.

Assume $L$ is a semisimple Hopf algebra of dimension 8. By [Mk1], $L$ is isomorphic to one of the following Hopf algebras:

- $\mathbb{k} C_{8}, \mathbb{k}\left(C_{2} \times C_{4}\right), \mathbb{k}\left(C_{2} \times C_{2} \times C_{2}\right)$ (abelian case),

- $\mathbb{k} Q_{8}, \mathbb{k} \mathbb{D}_{4}$, where $Q_{8}$ is the quaternion group (nonabelian case),

- $\mathbb{k}^{Q_{8}}$, with $\mathbb{k}^{Q_{8}}=\mathbb{k} C_{4} \oplus \mathcal{M}^{*}(2, \mathbb{k})$ as coalgebras (dual of non-abelian case),

- $\mathbb{k}^{\mathbb{D}_{4}}$, with $\mathbb{k}^{\mathbb{D}_{4}}=\mathbb{k} C_{4} \oplus \mathcal{M}^{*}(2, \mathbb{k})$ as coalgebras (dual of non-abelian case), or

- $B_{8}=\mathbb{k}\left(C_{2} \times C_{2}\right) \oplus \mathcal{M}^{*}(2, \mathbb{k})$ as coalgebras, see the definition in Remarks 3.7 and 3.8.

Also recall that every Hopf algebra of dimension $2 p$ is a group algebra or isomorphic to $\mathbb{k}^{\mathbb{D}_{p}}$, the dual of the group algebra on the dihedral group of order $2 p$.

Proposition 3.6. Let $H$ be a nonsemisimple nonpointed Hopf algebra of dimension $8 p$. If $H$ has the Chevalley property, then $\operatorname{dim} H_{0}=4 p$. 
Proof. Since $H$ is nonsemisimple and nonpointed, we have that $\operatorname{dim} H_{0}$ cannot be $1,2,4, p$ or $8 p$. Further, by Theorem 3.5, we also have that $\operatorname{dim} H_{0} \neq 8$. We show that $2 p$ is also impossible.

Assume $\operatorname{dim} H_{0}=2 p$. Since $H$ is not pointed, we must have that $H_{0} \cong \mathbb{R}^{\mathbb{D}_{p}}$. Then gr $H \cong R \# \mathbb{k}^{\mathbb{D}_{p}}$ with $R$ a connected graded Hopf algebra in ${ }_{\mathbb{k}^{\mathbb{D}} p}^{\mathbb{\mathbb { W }}_{p}} \mathcal{Y} \mathcal{D}$. Let $V=R(1)$ be the vector space of homogeneous elements of degree one and consider the subalgebra $\mathfrak{B}(V)$ of $R$ generated by $V$; it is a Nichols algebra of dimension less than or equal to 4 . Since $\mathbb{k}_{\mathbb{R} p}^{\mathbb{R}_{p}} \mathcal{Y} \mathcal{D} \simeq{\underset{\mathbb{D}}{p}}_{p} \mathcal{Y}$ as braided tensor categories, $\mathfrak{B}(V)$ corresponds to a Nichols algebra $\mathfrak{B}(W)$ of dimension less or equal than 4 in $\mathbb{D}_{p} \mathcal{Y} \mathcal{D}$. For dihedral groups $\mathbb{D}_{n}$ with $n$ odd, by [AHS, Theorem 4.8] the only possible finite-dimensional Nichols algebra over a simple module in ${\mathbb{\mathbb { D } _ { n }}}_{n} \mathcal{Y \mathcal { D }}$ comes from the conjugacy class $\left\{a, a y, a y^{2}, \ldots, a y^{n-1}\right\}$ in $\mathbb{D}_{n}$ and the braiding induced from the category. So, if $W \in{\underset{\mathbb{D}}{p}}_{p} \mathcal{Y} \mathcal{D}$ and $\mathfrak{B}(W)$ is finite-dimensional, then $W$ must contain a simple module isomorphic to this simple module of dimension $p$. As $\mathfrak{B}(W)$ is graded and the first two homogeneous components satisfy that $\mathfrak{B}(W)^{0}=\mathbb{k}, \mathfrak{B}(W)^{1}=W$, we have that $\operatorname{dim} \mathfrak{B}(W)>p$. But moreover, if the top degree of $\mathfrak{B}(W)$ is $m$, then by the Poincaré duality $\operatorname{dim} \mathfrak{B}(W)^{m-i}=\operatorname{dim} \mathfrak{B}(W)^{i}$. So, at least, $\operatorname{dim} \mathfrak{B}(W) \geq 2+p$, which is impossible.

In the remainder of this subsection we first recall the construction of a family of Hopf algebras of dimension $8 p$ due to $[\mathbf{C D M M}]$, and prove that any nonsemisimple, nonpointed and nonbasic Hopf algebra of dimension $8 p$ with the Chevalley property is isomorphic to a member of this family.

Remark 3.7. For $p \equiv 3 \bmod 4$ the semisimple Hopf algebras of dimension $4 p$ are:

- $\mathbb{k} \Gamma$ with $\Gamma$ an abelian group of order $4 p$;

- $\mathbb{k}_{\mathbb{D}_{2 p}}$ and $\mathbb{k}^{\mathbb{D}_{2 p}}$, where $\mathbb{k}^{\mathbb{D}_{2 p}} \cong \mathbb{k}\left[C_{2} \times C_{2}\right] \oplus \mathcal{M}^{*}(2, \mathbb{k})^{p-1}$ as coalgebras;

- $\mathbb{k} \operatorname{Dic}_{p}$ and $\mathbb{k}^{\mathrm{Dic}_{p}}$, where $\mathbb{k}^{\mathrm{Dic}} \cong \mathbb{k} C_{4} \oplus \mathcal{M}^{*}(2, \mathbb{k})^{p-1}$ as coalgebras;

- $A_{4 p}$, with $A_{4 p}=\mathbb{k} C_{4} \oplus \mathcal{M}^{*}(2, \mathbb{k})^{p-1}$ as coalgebras and $A_{4 p} \simeq A_{4 p}^{*}$ (non-trivial self-dual case);

- $B_{4 p}$, with $B_{4 p}=\mathbb{k}\left(C_{2} \times C_{2}\right) \oplus \mathcal{M}^{*}(2, \mathbb{k})^{p-1}$ as coalgebras and $B_{4 p} \simeq B_{4 p}^{*}$.

Here, $A_{4 p}$ denotes the $\mathbb{k}$-algebra generated by the elements $a, s_{+}$and $s_{-}$satisfying the relations

$$
a^{2}=1, \quad a s_{ \pm}=s_{ \pm} a, \quad s_{ \pm}^{2}=1, \quad\left(s_{+} s_{-}\right)^{p}=1 .
$$

Let $e_{0}=(1+a) / 2$ and $e_{1}=(1-a) / 2$. Then $A_{4 p}$ is a Hopf algebra with

$$
\begin{aligned}
& \Delta(a)=a \otimes a, \\
& \varepsilon(a)=1, \\
& \mathcal{S}(a)=a \\
& \Delta\left(s_{ \pm}\right)=s_{ \pm} \otimes e_{0} s_{ \pm}+s_{\mp} \otimes e_{1} s_{ \pm}, \\
& \varepsilon\left(s_{ \pm}\right)=1 \text {, } \\
& \mathcal{S}\left(s_{ \pm}\right)=e_{0} s_{ \pm}+e_{1} s_{\mp} .
\end{aligned}
$$

On the other hand, $B_{4 p}$ is the Hopf algebra defined in the same way, but replacing the last relation in (3.1) by $\left(s_{+} s_{-}\right)^{p}=a$. For more details, see [Mk2, Definition 3.3]. These Hopf algebras were first constructed by Gelaki, see [Ge1].

If $p \equiv 1 \bmod 4$ then there is an additional nonabelian group of order $4 p$ given by the semidirect product $\Gamma_{4 p}=C_{p} \rtimes_{\chi} C_{4}$, see Subsection 2.3. If $C_{p}=\langle y\rangle$ and $C_{4}=\langle x\rangle$ then $\chi(x)(y)=y^{(p-1) / 2}$.

REMARK 3.8. If $p=2$, the definition of $B_{4 p}$ above still makes sense and $B_{8}$ is the semisimple Hopf algebra of dimension 8 due to Kac and Paljutkin.

Let $N>1$ be an integer and $q \in \mathbb{k}$ a primitive $N$ th root of 1 . Recall that for $n \in \mathbb{N}$

$$
(n)_{q}=\frac{q^{n}-1}{q-1}, \quad(n)_{q} !=(n)_{q}(n-1)_{q} \cdots(1)_{q} .
$$

Let $R_{q}$ denote the graded algebra $\mathbb{k}[y] /\left(y^{N}\right)$ endowed with a coalgebra structure such that

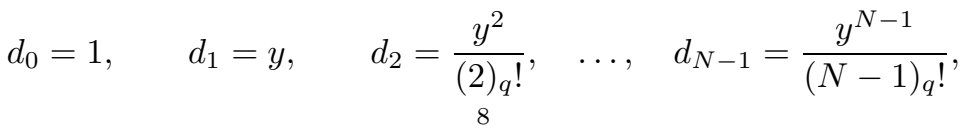


form a divided power sequence in the sense that $\Delta\left(d_{n}\right)=\sum_{i=0}^{n} d_{i} \otimes d_{n-i}$ for $0 \leq n<N$. The homogeneous component $R_{q}(n)$ is $\mathbb{k} y^{n}\left(=\mathbb{k} d_{n}\right)$ and is 0 if $n \geq N$.

Definition 3.9. [CDMM, Definition 2.1] A Yetter-Drinfeld (or YD) datum for $R_{q}$, is a triple $(L, g, \chi)$ which consists of a Hopf algebra $L$ with bijective antipode, a group-like element $g$ in $L$ and an algebra map $\chi: L \rightarrow \mathbb{k}$ such that $\chi(g)=q$ and

$$
(\chi \rightarrow h) g=g(h<\chi) \text { for all } h \in L .
$$

Using the YD-datum $(L, g, \chi)$ for $R_{q}$, one can define the $L$-action and $L$-coaction on $R_{q}$ by:

$$
h \cdot y^{n}=\chi^{n}(h) y^{n} \quad \text { and } \quad \delta\left(y^{n}\right)=g^{n} \otimes y^{n}, \quad \text { for } n=0, \ldots, N-1,
$$

so that $R_{q}$ is a graded braided Hopf algebra in ${ }_{L}^{L} \mathcal{Y D}$. Conversely, it can be seen that any structure that makes $R_{q}$ into a graded braided Hopf algebra in ${ }_{L}^{L} \mathcal{Y D}$ arises uniquely from a YD datum for $R_{q}$, see [AS2]. Note that by the above definition $q \neq 1$ and so $g \neq 1$. Thus to have a YD datum, it is necessary to have $g \neq 1$ in the center of $G(L)$.

EXAmples 3.10. (a) Let $\mathbb{k}^{\text {Dic }_{p}}$ be the function algebra on the dicyclic group of order $4 p$. By [CDMM], this commutative Hopf algebra is generated by the elements $a, x$ satisfying:

$$
\begin{array}{rlll}
a^{2}=1, & \varepsilon(a)=1, & \Delta(a)=a \otimes a, & \mathcal{S}(a)=a, \\
x^{2 p}=1, & \varepsilon(x)=1, & \Delta(x)=x \otimes e_{0} x+a x^{-1} \otimes e_{1} x, & \mathcal{S}(x)=e_{0} x^{-1}+e_{1} x .
\end{array}
$$

Here, $e_{0}=(1+a) / 2, e_{1}=(1-a) / 2$ and $g=\left(e_{0}+\sqrt{-1} e_{1}\right) x^{p}$ is a group-like element of order 4 that generates $G\left(\mathbb{k}^{\mathrm{Dic}_{p}}\right)$.

A YD-Datum for $R_{-1}=\mathbb{k}[y] /\left(y^{2}\right)$ is given by the triple $\left(\mathbb{k}^{\operatorname{Dic}_{p}}, g, \chi\right)$, where $\chi: \mathbb{k}^{\mathrm{Dic}_{p}} \rightarrow \mathbb{k}$ is the algebra map defined by $\chi(g)=-1, \chi(a)=1$ and $\chi(x)=-1$.

(b) Consider the semisimple Hopf algebra $A_{4 p}$ from Remark 3.7. There exist two YD-Datum for $R_{-1}=$ $\mathbb{k}[y] /\left(y^{2}\right)$, see [CDMM, Theorem 4.3], which are isomorphic to each other. They are given by the pairs $\left(s_{+}(p), \chi_{2}\right)$ and $\left(s_{+}(p) a, \chi_{3}\right)$, where $s_{+}(p)=s_{+} s_{-} s_{+} s_{-} \cdots(p$-times $) \in G\left(A_{4 p}\right)$ and $\chi_{2}, \chi_{3}$ are defined by $\chi_{2}(a)=-1, \chi_{2}\left(s_{ \pm}\right)=1$ and $\chi_{3}(a)=-1=\chi_{3}\left(s_{ \pm}\right)$.

Lemma 3.11. Let $H$ be a nonsemisimple Hopf algebra of dimension $2 n$ with antipode $\mathcal{S}$. Assume that $H$ has a semisimple Hopf subalgebra $K$ of dimension $n$. Then,

(a) $H$ has the Chevalley property with $K=H_{0}$, ord $\left(\mathcal{S}^{2}\right)=2$ and $n$ must be even.

(b) Consider the graded Hopf algebra gr $H=R \# K$. Then $\operatorname{dim} R=2, R \cong R_{-1}$ as $\mathbb{k}$-algebras, and $R$ admits a YD-datum structure $(K, g, \chi)$.

Proof. (a) Denote by $\pi: H^{*} \rightarrow K^{*}$ the adjoint of the inclusion of $K$ in $H$ and set $R=\left(H^{*}\right)^{c o \pi}$. Then by Proposition 2.5, $R=\mathbb{k}\{1, x\}$, with $x$ a $(1, g)$-primitive element, $g \in G\left(H^{*}\right)$ and $2 \mid \operatorname{ord}(g)$. If $x$ is trivial, then $H^{*}$ fits into an exact sequence of Hopf algebras $0 \rightarrow \mathbb{k} C_{2} \rightarrow H^{*} \rightarrow K^{*} \rightarrow 0$, which implies that $H^{*}$ is semisimple, a contradiction. Thus, $x$ must be nontrivial. In such a case, we know that $x^{2}=0$ and $4 \mid \operatorname{dim} H^{*}$. Thus, $n$ must be even.

Further, as $R$ is left-normal, it follows that $h_{1} r \mathcal{S}\left(h_{2}\right) \in R$ for all $h \in H^{*}$ and $r \in R$. Moreover, one has that $\varepsilon\left(h_{1} x \mathcal{S}\left(h_{2}\right)\right)=\varepsilon(x) \varepsilon(h)=0$. Since $R^{+}=\mathbb{k} x$, we conclude $h_{1} x \mathcal{S}\left(h_{2}\right) \in \mathbb{k} x$. Thus, $h x=h_{1} x \mathcal{S}\left(h_{2}\right) h_{3}=$ $c x h$ where $c$ is a scalar, implying $H^{*} x \subset x H^{*}$. But in such a case, $\left(R^{+} H^{*}\right)^{2}=\left(x H^{*}\right)^{2}=x H^{*} x H^{*}=$ $x^{2} H^{*}=0$ so $R^{+} H^{*} \subseteq J\left(H^{*}\right)$. As $K$ is semisimple, it holds that $J\left(H^{*}\right) \subset R^{+} H^{*}$, from which follows that $J\left(H^{*}\right)=R^{+} H^{*}$. Then, $K^{*} \cong H^{*} / R^{+} H^{*}$ and hence $K \cong\left(H^{*} / J\left(H^{*}\right)\right)^{*}=H_{0}$.

Since $H$ is nonsemisimple, $0=\operatorname{Tr}\left(\mathcal{S}^{2}\right)$. Moreover, as $\left.\mathcal{S}^{2}\right|_{K}=$ id, $\mathcal{S}^{2}$ induces a linear automorphism $\overline{\mathcal{S}}^{2}$ on $H / K$ and we have

$$
0=\operatorname{Tr}\left(\mathcal{S}^{2}\right)=\operatorname{Tr}\left(\left.\mathcal{S}^{2}\right|_{K}\right)+\operatorname{Tr}\left(\overline{\mathcal{S}}^{2}\right)=n+\operatorname{Tr}\left(\overline{\mathcal{S}}^{2}\right)
$$


Therefore, $\operatorname{Tr}\left(\overline{\mathcal{S}}^{2}\right)=-n$. Note that $\operatorname{dim}(H / K)=n$ and $\overline{\mathcal{S}}^{2}$ is of finite order. This forces $\overline{\mathcal{S}}^{2}=-$ id and so the eigenvalues of $\mathcal{S}^{2}$ are \pm 1 . Thus, we have $\mathcal{S}^{4}=$ id. Since $H$ is nonsemisimple, ord $\left(\mathcal{S}^{2}\right)=2$.

(b) Since $H$ is not semisimple, gr $H$ is not semisimple. Moreover, as $R=(\operatorname{gr} H)^{\operatorname{co} \pi}$ with $\pi: \operatorname{gr} H \rightarrow K$ the canonical projection, we have that $\operatorname{dim} R=2$. Then by Proposition $2.5, R$ has basis $\{1, x\}$ where $x$ is a nontrivial $(1, g)$-primitive and $x^{2}=0$. Then we must have that $R \simeq R_{-1}$ and $x \in R(1)$. Since $R(1) \in{ }_{K}^{K} \mathcal{Y D}$, there exists an algebra map $\chi: K \rightarrow \mathbb{k}$ such that

$$
h \cdot x=\chi(h) x \text { for all } h \in K .
$$

Then by [AS2, Lemma 8.1] and the following remark we have that $(K, g, \chi)$ is a YD-Datum for $R_{-1}$ (see Definition 3.9).

Definition 3.12. (cf. [CDMM]) Define $\mathcal{H}_{8 p}$ as the $\mathbb{k}$-algebra generated by the elements $a, x, z$ satisfying the relations

$$
\begin{aligned}
& a^{2}=1, \\
& x^{2 p}=1, \\
& z^{2}=a-1, \\
& a x=x a, \\
& a z=z a, \\
& x z=-z x \text {. }
\end{aligned}
$$

It is a Hopf algebra with the coalgebra structure and antipode given by

$$
\begin{aligned}
& \Delta(a)=a \otimes a, \quad \Delta(x)=x \otimes e_{0} x+a x^{-1} \otimes e_{1} x, \quad \Delta(z)=g \otimes z+z \otimes 1 \\
& \mathcal{S}(a)=a, \quad \mathcal{S}(x)=e_{0} x^{-1}+e_{1} x, \quad \mathcal{S}(z)=-g^{-1} z \\
& \varepsilon(a)=1, \quad \varepsilon(x)=1, \quad \varepsilon(z)=0,
\end{aligned}
$$

where $g=\left(e_{0}+\sqrt{-1} e_{1}\right) x^{p}$. Clearly, $\mathcal{H}_{8 p}$ contains $\mathbb{k}^{\text {Dic }_{p}}$ as a Hopf subalgebra and $g$ is a group-like element of order 4 that generates $G\left(\mathcal{H}_{8 p}\right)$. Moreover, by [CDMM, Theorem 4.2] we have that $\mathcal{H}_{8 p}$ is a lifting of a two-dimensional Nichols algebra over $\mathbb{k}^{\mathrm{Dic}_{p}}$.

REMARK 3.13. Note that the definition above is denoted $\mathcal{H}_{8 p}(1)$ in [CDMM]. There, the authors define the Hopf algebra $\mathcal{H}_{8 p}(\alpha)$ as above except that $z^{2}=\alpha(a-1)$. Since we are working over an algebraically closed field of characteristic $0, \mathcal{H}_{8 p}(\alpha)$ is isomorphic to $\mathcal{H}_{8 p}(1)$ for $\alpha \neq 0$, and we denote $\mathcal{H}_{8 p}(1)$ simply by $\mathcal{H}_{8 p}$. Notice that $\mathcal{H}_{8 p}(0) \simeq R_{-1} \# \mathbb{k}^{\text {Dic }_{p}}$.

Proposition 3.14. The Hopf algebra $\mathcal{H}_{8 p}$ is a nonsemisimple, nonpointed, nonbasic Hopf algebra of

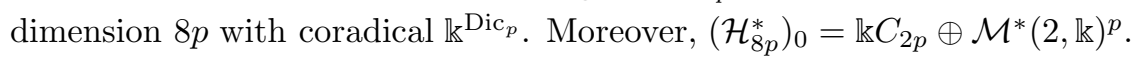

Proof. By [CDMM, Theorem 4.2], $\mathcal{H}_{8 p}$ is a non-trivial lifting of a quantum line $R_{-1}$ over the semisimple Hopf algebra $\mathbb{k}^{\mathrm{Dic}_{p}}$. Hence it is nonsemisimple and nonpointed. Since the diagram $R_{-1}$ is given by the YD-Datum $\left(\mathbb{k}^{\mathrm{Dic}_{p}}, g, \chi\right)$, with $\chi: \mathbb{k}^{\mathrm{Dic}_{p}} \rightarrow \mathbb{k}$ an algebra map defined by $\chi(g)=-1, \chi(a)=1$ and $\chi(x)=-1$, we have that $\operatorname{dim} R_{-1}=2$ and consequently, $\operatorname{dim} \mathcal{H}_{8 p}=8 p$.

To finish the proof, we find all simple representations of $\mathcal{H}_{8 p}$. Since not all simple representations are one-dimensional, the algebra is nonbasic.

Let $\xi$ be a primitive $2 p$ th root of unity. Since $\mathbb{k}^{\mathrm{Dic}_{p}}$ is a commutative algebra, all simple representations are one-dimensional. They are given by the family of $\mathbb{k}$-vector spaces $\left(V_{i, j}=\mathbb{k} v_{i, j}\right)_{i \in \mathbb{Z}_{2}, j \in \mathbb{Z}_{2 p}}$ with the action determined by $a \cdot v_{i, j}=(-1)^{i} v_{i, j}$ and $x \cdot v_{i, j}=\xi^{j} v_{i, j}$.

The one-dimensional representations of $\mathcal{H}_{8 p}$ are given by the vector spaces $W_{j}=\mathbb{k} w_{j}$ with $j \in \mathbb{Z}_{2 p}$. The action is determined by $a \cdot w_{j}=w_{j}, x \cdot w_{j}=\xi^{j} w_{j}$ and $z \cdot w_{j}=0$. Clearly, $W_{j} \simeq W_{\ell}$ if and only if $\ell=j$ in $\mathbb{Z}_{2 p}$. 
The two-dimensional representations of $\mathcal{H}_{8 p}$ are given by the vector spaces $U_{i}=\mathbb{k} u_{1}^{i}+\mathbb{k} u_{2}^{i}$ with $i \in \mathbb{Z}_{2 p}$. The action is determined by

$$
a \cdot u_{j}^{i}=-u_{j}^{i} \quad x \cdot u_{j}^{i}=(-1)^{j-1} \xi^{i} u_{j}^{i}, \quad z \cdot u_{1}^{i}=u_{2}^{i}, \quad \text { and } \quad z \cdot u_{2}^{i}=-2 u_{1}^{i},
$$

for $j=1,2$. We show now that $U_{i}$ is simple for all $i \in \mathbb{Z}_{2 p}$ and $U_{i} \simeq U_{j}$ if and only if $i=j$ or $i=j+p$ in

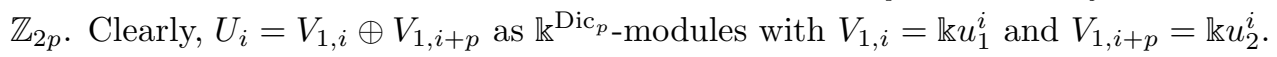

Let $i \in \mathbb{Z}_{2 p}$ and assume that $U_{i}$ is not simple. Since it is two-dimensional, it must contain a onedimensional simple module, say spanned by $v=\beta u_{1}^{i}+\gamma u_{2}^{i}$. Thus, $a \cdot v=-v$ and $x \cdot v=\mu v$ for some $2 p$ th root of unity $\mu$. Since $x \cdot v=\beta \xi^{i} u_{1}^{i}-\gamma \xi^{i} u_{2}^{i}$, it follows that $\mu=\xi^{i}$ and $v=\beta u_{1}^{i}$ or $\mu=-\xi^{i}=\xi^{i+p}$ and $v=\gamma u_{2}^{i}$. But this is impossible, because $z$ would not stabilize $v$.

Let $\varphi: U_{i} \rightarrow U_{j}$ be a morphism of $\mathcal{H}_{8 p}$-modules. Since both modules are simple, we have that $\varphi=0$ or $\varphi$ is an isomorphism. Assume that $\varphi \neq 0$. Then $\varphi\left(u_{1}^{i}\right)$ must be an eigenvector of $x$ in $U_{j}$ of eigenvalue $\xi^{i}$, since the eigenvalues of $x$ in $U_{j}$ are $\pm \xi^{j}$, the claim follows. Consequently, we have at least $p$ isomorphism classes of two-dimensional simple modules.

Then $\operatorname{dim}\left(\mathcal{H}_{8 p}^{*}\right)_{0} \geq 6 p$ with group-likes of order at least $2 p$. By [BG, Proposition 3.2], $\mathcal{H}_{8 p}^{*}$ has a nontrivial skew-primitive element and so the dimension of the linear space spanned by the nontrivial skewprimitive elements is at least $2 p$. Thus, $\left(\mathcal{H}_{8 p}^{*}\right)_{0}=\mathbb{k} C_{2 p} \oplus \mathcal{M}^{*}(2, \mathbb{k})^{p}$ as claimed.

Theorem 3.15. Let $H$ be a nonsemisimple, nonpointed, nonbasic Hopf algebra of dimension $8 p$ with the Chevalley property. Then $H$ is isomorphic either to $R_{-1} \# A_{4 p}$ or $\mathcal{H}_{8 p}$.

Proof. By Proposition 3.6, we know that $\operatorname{dim} H_{0}=4 p$. If we denote $A=\operatorname{gr} H$, then we have that $A=R \# H_{0}$ and by Lemma $3.11, R=R_{-1}$ admits a YD-datum given by $\left(H_{0}, g, \chi\right)$ for some $g \in G\left(H_{0}\right)$ and $\chi: H_{0} \rightarrow \mathbb{k}$. If $p \equiv 3 \bmod 4$, we have four possibilities (up to isomorphism) for $H_{0}: A_{4 p}, B_{4 p}, \mathbb{k}^{\mathbb{D}_{2 p}}$ and $\mathbb{k}^{\mathrm{Dic}_{p}}$. If $p \equiv 1 \bmod 4$, there is an additional possibility given by $\mathbb{k}^{\Gamma_{4 p}}$.

First we show that the cases $B_{4 p}, \mathbb{k}^{\mathbb{D}_{2 p}}, \mathbb{k}^{\Gamma_{4 p}}$ are impossible. By [CDMM, Theorem 4.4], there is no YD-datum for $R$ when $H_{0}=B_{4 p}$ and so the first case is impossible. For $H_{0}=\mathbb{k}^{\mathbb{D}_{2 p}}$, by [CDMM, Theorem 4.1] there is one YD-datum but it admits no lifting. This implies that $H=A=R_{-1} \# \mathbb{R}^{\mathbb{D}_{2 p}}$ from which follows that $H^{*} \simeq R_{-1}^{*} \# \mathbb{k} \mathbb{D}_{2 p}$ is pointed, a contradiction. Finally, let $H_{0}=\mathbb{k}^{\Gamma_{4 p}}$ and assume that there exists a braided Hopf algebra $R$ of dimension 2 such that $A=R \# \mathbb{k}^{\Gamma_{4 p}}$. Then $A^{*} \simeq R^{*} \# \mathbb{k} \Gamma_{4 p}$, where $R^{*}$ is a braided Hopf algebra in $\Gamma_{\Gamma_{4} p} \mathcal{Y D}$. Since this category is semisimple, $R^{*}$ is a sum of simple modules. But since the simple modules in ${ }_{\Gamma_{4 p}}^{\Gamma_{4 p}} \mathcal{Y D}$ have dimensions 1,4 or $p$ by Table $1, R^{*}$ must be the sum of two simple one-dimensional modules of the form $M(\mathcal{O}, \rho)$ with $\mathcal{O}=\{1\}$. This is impossible, because $R^{*}$ would contain the Nichols algebra generated by these modules and the later are infinite-dimensional by Lemma 2.6.

If $H_{0}=A_{4 p}$, then by [CDMM, Theorem 4.3] $R$ admits a unique (up to isomorphism) YD-datum and it has no lifting. Thus $H=A=R_{-1} \# A_{4 p}$; in this case, $H$ is a self-dual Hopf algebra. If $H_{0}=\mathbb{k}^{\mathrm{Dic}_{p}}$, then by [CDMM, Theorem 4.2], $A=R_{-1} \# \mathbb{k}^{\mathrm{Dic}_{p}}$ has a nontrivial lifting isomorphic to $\mathcal{H}_{8 p}$.

We end this section with the classification of Hopf algebras of dimension $8 p$ with the Chevalley property. The determination of the pointed Hopf algebras in this case follows essentially from the results of Nichols, Graña ([Ni], [Gr1, Gr2]), and several authors that contributed to the solution of the abelian case, among them Andruskiewitsch, Schneider, Heckenberger and Angiono, see for example [AA].

Theorem 3.16. Let $H$ be a nonsemisimple Hopf algebra of dimension $8 p$ with the Chevalley property.

(a) Assume $H$ is pointed. Then $H$ is isomorphic to one of the Hopf algebras constructed in [Gr1].

(b) Assume neither $H$ nor $H^{*}$ is pointed. Then $H$ is isomorphic either to $R_{-1} \# A_{4 p}$ or $\mathcal{H}_{8 p}$. 
Proof. (a) Assume $H$ is pointed. Then $|G(H)| \in\{2,4,8,2 p, 4 p\}$. The cases $|G(H)|=2$ and 8 are impossible by [Ni, Theorem 4.2.1] and [Gr1, Corollary 4.1], respectively, because $\operatorname{dim} H$ would be a power of 2. The case $|G(H)|=4$ is also impossible for the same reason, although the result is due to several authors working on pointed Hopf algebras over abelian groups. For details see for example [AA]. Finally, if $|G(H)|=2 p$ or $4 p$, then $H$ is isomorphic to one of the Hopf algebras constructed in [Gr1], since $\frac{\operatorname{dim} H}{|G(H)|}<32$.

(b) Assume $H$ is neither pointed nor basic. Then $H$ is isomorphic either to $R_{-1} \# A_{4 p}$ or $\mathcal{H}_{8 p}$ by Theorem 3.15 .

\section{Nonsemisimple Hopf algebras of dimension 24}

We end the paper with some structural results on nonsemisimple Hopf algebras of dimension 24 . Throughout this section $H$ will be a nonsemisimple Hopf algebra of dimension 24. We begin with the following lemma.

Lemma 4.1. If $G(H)$ is nontrivial, then $H$ has a nontrivial skew-primitive element.

Proof. It suffices to prove the same statement for $H^{*}$. Note that $H^{*}$ having the Chevalley property is equivalent to the $\mathbb{k}$-linear full subcategory generated by the simple $H$-modules being a fusion subcategory of $H$-mod.

Suppose $G\left(H^{*}\right)$ is nontrivial and $H^{*}$ has only trivial skew-primitive elements. Then $H$ has a nontrivial 1-dimension $H$-module, and $\operatorname{Ext}\left(\mathbb{k}_{,} \mathbb{k}_{\beta}\right)=\operatorname{Ext}\left(\mathbb{k}_{\beta}, \mathbb{k}\right)=0$ for all $\beta \in G\left(H^{*}\right)$. Thus, $P(\mathbb{k}) / J^{2} P(\mathbb{k})$ has a simple $H$-submodule $V$ such that $\operatorname{dim} V \geq 2$.

(i) We first observe that $\operatorname{dim} V=2$ otherwise $\operatorname{dim} V \geq 3$ and so

$$
\operatorname{dim} H \geq\left|G\left(H^{*}\right)\right| \operatorname{dim} P(\mathbb{k})+\operatorname{dim} V \operatorname{dim} P(V) \geq 2 \cdot 5+3 \cdot 7>24 .
$$

(ii) We claim that if $W$ is another simple $H$-module with $\operatorname{dim} W>1$, then $W$ is projective with $\operatorname{dim} W=2$. Since

$\operatorname{dim} H \geq\left|G\left(H^{*}\right)\right| \operatorname{dim} P(\mathbb{k})+\operatorname{dim} V \operatorname{dim} P(V)+\operatorname{dim} W \operatorname{dim} P(W) \geq 2 \cdot 4+2 \cdot 5+\operatorname{dim} W \operatorname{dim} P(W)$.

This forces $\operatorname{dim} W \operatorname{dim} P(W) \leq 6$, and hence $\operatorname{dim} W=\operatorname{dim} P(W)=2$. In particular, $W$ is projective.

(iii) Since $V$ is the unique simple $H$-module with $\operatorname{dim} V>1$ that is not projective, $V^{*} \cong V$ and $\mathbb{k}_{\beta} \otimes V \cong V \cong V \otimes \mathbb{k}_{\beta}$ for all $\beta \in G\left(H^{*}\right)$. Since $P\left(\mathbb{k}_{\beta}\right) \cong \mathbb{k}_{\beta} \otimes P(\mathbb{k}), V$ is a simple $H$-submodule of $P\left(\mathbb{k}_{\beta}\right) / J^{2} P\left(\mathbb{k}_{\beta}\right)$. This implies that $\sum_{\beta \in G\left(H^{*}\right)} \mathbb{k}_{\beta}$ is an $H$-submodule of $P(V) / J^{2} P(V)$, and hence

$$
\operatorname{dim} P(V) \geq\left|G\left(H^{*}\right)\right|+2 \operatorname{dim} V=\left|G\left(H^{*}\right)\right|+4 \geq 6 .
$$

(iv) $\left|G\left(H^{*}\right)\right|=2$ for otherwise $\operatorname{dim} P(V) \geq 7$ and so

$$
\operatorname{dim} H \geq\left|G\left(H^{*}\right)\right| \operatorname{dim} P(\mathbb{k})+2 \operatorname{dim} P(V) \geq 3 \cdot 4+2 \cdot 7>24 .
$$

(v) We now claim that $V$ is the unique simple $H$-module with $\operatorname{dim} V>1$. Suppose not. By (ii), there is a 2-dimensional simple projective $H$-module $W$. The inequality

$$
\operatorname{dim} H \geq 2 \operatorname{dim} P(\mathbb{k})+2 \operatorname{dim} P(V)+(\operatorname{dim} W)^{2} \geq 2 \cdot 4+2 \cdot 6+2 \cdot 2=24
$$

implies that $W$ is the unique simple projective $H$-module,

$$
\operatorname{dim} P(\mathbb{k})=4 \text { and } \operatorname{dim} P(V)=6 .
$$

Therefore, $W \cong W^{*}$ and $\mathbb{k}_{\alpha} \otimes W \cong W \cong W \otimes \mathbb{k}_{\alpha}$, where $\alpha \in G\left(H^{*}\right)$ is the nontrivial group-like element. Since $W$ is projective, so is $W \otimes W^{*}$. Therefore, $W \otimes W^{*}$ is a direct sum of indecomposable projective $H$-modules. However, $W \otimes W^{*}$ maps surjectively onto $\mathbb{k}$ and $\mathbb{k}_{\alpha}$ which implies $P(\mathbb{k}) \oplus P\left(\mathbb{k}_{\alpha}\right)$ is a summand of $W \otimes W^{*}$. This is absurd as $\operatorname{dim}\left(W \otimes W^{*}\right)=\operatorname{dim} P(\mathbb{k})=\operatorname{dim} P\left(\mathbb{k}_{\alpha}\right)=4$. 
(vi) Let $m$ be the multiplicity of $V$ as a composition factor of $P(\mathbb{k})$. Then, by (iii), $m$ is also the multiplicity of $V$ as a composition factor of $P\left(\mathbb{k}_{\alpha}\right)$. Since $\mathbb{k}$ is algebraically closed of characteristic zero, $\operatorname{dim} P(V)$ is the multiplicity of $V$ in the composition factors of $H$ (cf. [CR, Theorem 54.19]). By (v), we have

$$
H=P(\mathbb{k}) \oplus P\left(\mathbb{k}_{\alpha}\right) \oplus 2 P(V) .
$$

As $V$ is composition factor of $P(\mathbb{k})$, we have that $m>0$. Since $\operatorname{dim} \operatorname{Soc}(P(V))=\operatorname{dim} V=2, \operatorname{Soc}(P(V))=V$ and hence $[P(V): V] \geq 2$. Therefore, $\operatorname{dim} P(V) \geq 2 m+4$. Thus, the inequality

$$
\operatorname{dim} H=2 \operatorname{dim} P(\mathbb{k})+2 \operatorname{dim} P(V) \geq 2(2+2 m)+2(2 m+4)=4(2 m+3)
$$

forces $m=1$. In particular, $\operatorname{dim} P(\mathbb{k})=4$. Now we have

$$
\operatorname{dim} H=2 \cdot 4+2 \operatorname{dim} P(V)
$$

and so $\operatorname{dim} P(V)=8$.

(vii) Since $P\left(\mathbb{k}_{\beta}\right) \otimes V$ is projective for any $\beta \in G\left(H^{*}\right)$ and it maps surjectively onto $V, P(V)$ is a summand of $P\left(\mathbb{k}_{\beta}\right) \otimes V$. This implies $P\left(\mathbb{k}_{\beta}\right) \otimes V \cong P(V)$ by comparing dimensions. Similarly, $V \otimes P\left(\mathbb{k}_{\beta}\right) \cong P(V)$.

(viii) We now prove that $H^{*}$ having the Chevalley property means that the $\mathbb{k}$-linear abelian full subcategory generated by $\mathbb{k}, \mathbb{k}_{\alpha}$ and $V$ is closed under the tensor product of $H$-mod. Since

$$
\operatorname{dim} \operatorname{Hom}_{H}\left(P\left(\mathbb{k}_{\beta}\right), V \otimes V\right)=\operatorname{dim} \operatorname{Hom}_{H}\left(V^{*} \otimes P\left(\mathbb{k}_{\beta}\right), V\right)=\operatorname{dim}_{\operatorname{Hom}}(P(V), V)=1,
$$

the multiplicity of the $\mathbb{k}_{\beta}$ as a composition factor of $V \otimes V$ is 1 . The dual basis map db $: \mathbb{k} \rightarrow V \otimes V^{*}$ and the evaluation map ev : $V^{*} \otimes V \rightarrow \mathbb{k}$ are nonzero $H$-module maps, so are $\mathbb{k}_{\beta} \otimes \mathrm{db}$ and $\mathbb{k}_{\beta} \otimes$ ev for all $\beta \in G\left(H^{*}\right)$. Therefore, both $\mathbb{k}$ and $\mathbb{k}_{\alpha}$ are summands of $V \otimes V$, and hence $V \otimes V \cong V \oplus \mathbb{k} \oplus \mathbb{k}_{\alpha}$. By (iii), the $\mathbb{k}$-linear abelian full subcategory $\mathcal{D}$ generated by $\mathbb{k}, \mathbb{k}_{\alpha}$ and $V$ is a fusion subcategory of $H$-mod.

(ix) By the reconstruction theorem, $\mathcal{D}$ is monoidally equivalent to $\bar{H}$-mod for some semisimple quotient Hopf algebra $\bar{H}$ of $H$. Since $\operatorname{dim} \bar{H}=1+1+2^{2}=6$, it follows from the classification of 6 -dimensional Hopf algebras that $\bar{H} \cong \mathbb{k S}_{3}$. Thus, $\mathbb{K}^{\mathbb{S}_{3}} \cong\left(H^{*}\right)_{0}$ but this contradicts that the smallest Hopf algebras with the Chevalley property and the coradical isomorphic to $\mathbb{k}^{\mathbb{S}_{3}}$ is of dimension $72[\mathbf{A V}]$. This completes the proof of this lemma.

Corollary 4.2. If $H$ and $H^{*}$ do not have the Chevalley property, then both $H$ and $H^{*}$ have a nontrivial skew-primitive element.

Proof. By [BG, Lemma 4.25], $|G(H)|$ and $\left|G\left(H^{*}\right)\right|$ are even. The statement then follows immediately from Lemma 4.1.

Proposition 4.3. Let $H$ be a nonbasic Hopf algebra of dimension 24 without the Chevalley property. Then $H$ is generated by its coradical.

Proof. Let $K$ be the Hopf subalgebra of $H$ generated by the coradical $H_{0}$. As $H$ is nonpointed, $\operatorname{dim} H_{0}>4$. Since 6 -dimensional Hopf algebras are semisimple, $\operatorname{dim} K=8,12$ or 24 . Since $H$ does not have the Chevalley property, then $K \neq H_{0}$ and $K$ is nonsemisimple and nonpointed. Consider the standard filtration associated to $K$ and write gr $H=R \# K$, see $[\mathbf{A C}]$.

Assume $\operatorname{dim} K=8$. In such a case, $\operatorname{dim} R=3$. Let $V$ be an indecomposable Yetter-Drinfeld submodule of $R(1)$ and consider the subalgebra $L$ of $R$ generated by $V$. Note that $L$ is not necessarily a Nichols algebra since it might contain primitive elements in degree bigger than one. In any case, there is a projection $L \rightarrow \mathfrak{B}(V)$ and consequently $\operatorname{dim} L \geq \operatorname{dim} \mathfrak{B}(V)$. This implies by [GG, Theorem A] that $V$ is simple and $\operatorname{dim} V=1$ or 2 . The latter case is impossible since $\operatorname{dim} \mathfrak{B}(V)>3$. In the former case, by [GG, Proposition 
3.2] it follows that $\operatorname{dim} L=2$, which is impossible, since gr $H$ would contain a Hopf subalgebra of dimension 16.

Assume now that $\operatorname{dim} K=12$. In such a case, $\operatorname{dim} R=2$. By the classification result of Natale [Na] (see also $[\mathbf{C N}]$ ), there is a unique Hopf algebra (up to isomorphism) whose coradical is not a Hopf subalgebra. It is the dual of a pointed Hopf algebra; denote it by $\mathcal{C}$. As before, let $V$ be an indecomposable YetterDrinfeld submodule of $R(1)$ and consider the subalgebra $L$ of $R$ generated by $V$. Since $\operatorname{dim} R(0)=1$ and so $\operatorname{dim} R(1)=1$. Let $x$ be a nonzero element of $R(1)$. Then $x^{2}=0$ and so $R \cong R_{-1}$. In particular, $R^{*} \cong R$ as algebras and coalgebras. Thus by $[\mathbf{X}$, Theorem $\mathrm{B}], H$ is isomorphic to bosonization $\bigwedge \mathbb{k}_{\chi} \# \mathcal{C}$. This implies that $H^{*} \simeq \bigwedge \mathbb{k}_{\chi} \# \mathcal{C}^{*}$ and whence $H$ would be basic, a contradiction.

\section{References}

[A] N. Andruskiewitsch An Introduction to Nichols Algebras. In Quantization, Geometry and Noncommutative Structures in Mathematics and Physics. A. Cardona, P. Morales, H. Ocampo, S. Paycha, A. Reyes, eds., pp. 135-195, Springer (2017).

[AA] N. Andruskiewitsch and I. Angiono, On Finite dimensional Nichols algebras of diagonal type, Bull. Math. Sci. (2017) 7, 353-573.

[AC] N. Andruskiewitsch and J. Cuadra, On the structure of (co-Frobenius) Hopf algebras, J. Noncommutative Geometry 7 (2013), Issue 1, pp. 83-104.

[AG] N. Andruskiewitsch and M. Graña, From racks to pointed Hopf algebras, Adv. Math. 178 (2003), $177-243$.

[AHS] N. Andruskiewitsch, I. Heckenberger and H.J. Schneider, The Nichols algebra of a semisimple Yetter-Drinfeld module, Am. J. Math. 1326 (2010) pp. 1493-1547.

[AN] N. Andruskiewitsch and S. NATAle, Counting arguments for Hopf algebras of low dimension, Tsukuba Math J. 25, No. 1 (2001), 187-201.

[AS1] N. Andruskiewitsch and H.-J. Schneider, Lifting of quantum linear spaces and pointed Hopf algebras of order $p^{3}$, J. Algebra 209 (1998), no. 2, 658-691.

[AS2] N. Andruskiewitsch and H.-J. Schneider, Hopf algebras of order $p^{2}$ and braided Hopf algebras of order $p, J$. Algebra 199 (1998), no. 2, 430-454.

[AV] N. Andruskiewitsch and C. VAy, Finite dimensional Hopf algebras over the dual group algebra of the symmetric group in three letters, Comm. Algebra 39 (2011), 4507-4517.

[B] M. Beattie, An isomorphism theorem for Ore extension Hopf algebras. (English summary) Comm. Algebra 28 (2000), no. 2, 569-584.

[BDG] M. Beattie, S. DăsCăLescu and L. Grünenfelder, On the number of types of finite-dimensional Hopf algebras, Invent. Math. 136 (1999), no. 1, 1-7.

[BG] M. Beattie and G.A. García, Techniques for classifying Hopf algebras and applications to dimension $p^{3}$, Comm. Algebra, 41 (2013), 3108-3129

[BG2] Classifying Hopf algebras of a given dimension. Contemp. Math. 585 (2013), 125-152.

[CDMM] C. Calinescu, S. DĂSCĂLESCU, A. Masuoka, C. Menini, Quantum lines over non-cocommutative cosemisimple Hopf algebras, J. Algebra 273 (2004), 753-779.

[CN] Y-L. Cheng and S-H. NG, On Hopf algebras of dimension 4p, J. Algebra 328 (2011), 399-419.

[CR] C. W. CuRTIS and I. ReINER, Representation theory of finite groups and associative algebras, Interscience Publishers, New York-London, Pure and Applied Mathematics, Vol. XI,1962.

[EG] P. Etingof and S. Gelaki, On families of triangular Hopf algebras, Int. Math. Res. Not. (2002), no. 14, 757-768.

[GG] G. A. García and J. M. J. Giraldi, On Hopf algebra over quantum subgroups. Preprint: arXiv:1605. 03995.

[GV] G.A. García and C. VAY, Hopf algebras of dimension 16, Algebr. Represent. Theory, 13 (2010), no. 4, 383-405..

[Ge1] S. Gelaki, Quantum groups of dimension $p q^{2}$, Israel J. Math. 102 (1997), 227-267.

[Ge2] _ Pointed Hopf algebras and Kaplansky's 10th conjecture, J. Algebra 209 (1998), no. 2, 635-657.

[Gr1] M. GrañA, On Nichols algebras of low dimension, in "New Trends in Hopf Algebra Theory"; Contemp. Math. 267 (2000), 111-136.

[Gr2] A A freeness theorem for Nichols algebras, J. Algebra 231 (2000), 235-257.

[Gr3] Pointed Hopf algebras of dimension 32. (English summary) Comm. Algebra 28 (2000), no. 6, 2935-2976. 
[Gr4] W Finite dimensional Nichols algebras of non-diagonal group type, zoo of examples available at \protect \vrulewidth0pthttp://mate.dm.uba.ar/ matiasg/zoo.html .

[H] I. Heckenberger, Classification of arithmetic root systems, Adv. Math. 220 (2009), 59-124.

[HN] M. Hilgemann and S.-H. NG, Hopf algebras of dimension 2p2 , J. Lond. Math. Soc. (2) 80 (2009), no. 2, 295-310.

[IK] M. IzUmi and H. KosAKI, Kac algebras arising from composition of subfactors: general theory and classification, Mem. Amer. Math. Soc. 158 (2002), no. 750, 198 pp.

[K] I. Kaplansky, Bialgebras, Department of Mathematics, University of Chicago, Chicago, Ill., 1975, Lecture Notes in Mathematics.

[Ma] S. Majid, Cross Products by Braided Groups and Bosonization, J. Algebra 163, no.1, 165-190.

[Mk1] A. Masuoka, Semisimple Hopf algebras of dimension 6, 8, Israel J. Math. 92 (1995), 361-373.

[Mk2] Cocycle deformations and Galois objects for some cosemisimple Hopf algebras of finite dimension, Contemp. Math. 267 (2000) 195-214.

[Mo] S. Montgomery, Hopf Algebras and their Actions on Rings, CMBS Reg. Conf. Ser. in Math. 82, Amer. Math. Soc., 1993.

[Na] S. Natale, Hopf algebras of dimension 12, Algebr. Represent. Theory 5 (5) (2002), 445-455.

$[\mathrm{Ng}] \quad$ S-H. NG, Hopf algebras of dimension pq, II, J. Algebra 319, no. 7 (2008), 2772-2788.

[Ni] W. Nichols, Bialgebras of type one. Comm. Algebra 6, no. 15 (1978), 1521-1552.

[NN] C. Negron and S-H. NG, Gauge invariants from the powers of antipodes, Pacific J. Math. 291 (2017), no. 2, 439-460.

[Ra] D.E. RAdFord, The structure of Hopf algebras with a projection, J. Algebra 92 (1985), no.2, 322-347.

[RS] D. E. RADFORD and H-J. Schneider, On the even powers of the antipode of a finite-dimensional Hopf algebra, J. Algebra 251 (2002), 185-212.

[Ş97] D. ŞTEFAn, The set of types of $n$-dimensional semisimple and cosemisimple Hopf algebras is finite, J. Algebra 193 (2) (1997), 571-580.

[S] M. Sweedler, Hopf algebras, Benjamin, New York, (1969).

[X] R. XIONG, On Hopf algebras over the unique 12-dimensional Hopf algebra without the dual Chevalley property. Preprint: arXiv: 1712.00826.

Mount Allison University, Sackville, NB E4L 1E6, Canada

E-mail address: mbeattie@mta.ca

Departamento de Matemática, Facultad de Ciencias Exactas, Universidad Nacional de la Plata. COnicet. Casilla de Correo 172, (1900) La Plata, Argentina

E-mail address: ggarcia@famaf.unc.edu.ar

Department of Mathematics, Louisiana State University, Baton Rouge, LA 70803, USA

E-mail address: rng@math.lsu.edu

J.R.: Mathematics Department, SUNY Cortland, Cortland, NY 13045, USA

E-mail address: jolie.roat@cortland.edu 